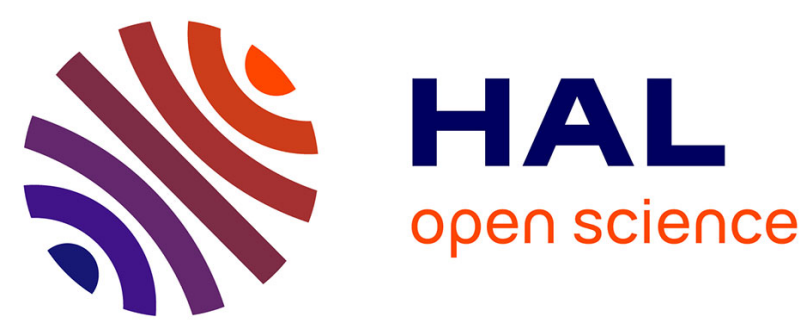

\title{
MANET network management and performance monitoring for NHDP and OLSRv2
}

\author{
Ulrich Herberg, Thomas Heide Clausen, Robert Cole
}

\section{To cite this version:}

Ulrich Herberg, Thomas Heide Clausen, Robert Cole. MANET network management and performance monitoring for NHDP and OLSRv2. 2010 International Conference on Network and Service Management (CNSM), Oct 2010, Niagara Falls, Canada. pp.290-293, 10.1109/CNSM.2010.5691209 . hal-02263423

HAL Id: hal-02263423

https://hal-polytechnique.archives-ouvertes.fr/hal-02263423

Submitted on 4 Aug 2019

HAL is a multi-disciplinary open access archive for the deposit and dissemination of scientific research documents, whether they are published or not. The documents may come from teaching and research institutions in France or abroad, or from public or private research centers.
L'archive ouverte pluridisciplinaire HAL, est destinée au dépôt et à la diffusion de documents scientifiques de niveau recherche, publiés ou non, émanant des établissements d'enseignement et de recherche français ou étrangers, des laboratoires publics ou privés. 


\section{MANET Network Management and Performance Monitoring for NHDP and OLSRv2}

\author{
Ulrich Herberg, Thomas Clausen \\ Hipercom@LIX \\ Ecole Polytechnique \\ France \\ Email: ulrich@herberg.name, thomas@thomasclausen.org
}

\author{
Robert Cole \\ Space and Terrestrial Communications \\ US Army CERDEC \\ Aberdeen Proving Ground, MD, USA 21005 \\ robert.g.cole@us.army.mil
}

\begin{abstract}
Mobile Ad Hoc NETworks (MANETs) are generally thought of as infrastructureless and largely "un-managed" network deployments, capable of accommodating highly dynamic network topologies. Yet, while the network infrastructure may be "un-managed", monitoring the network performance and setting configuration parameters once deployed, remains important in order to ensure proper "tuning" and maintenance of a MANET. This paper describes a management framework for the MANET routing protocol OLSRv2, and its constituent protocol NHDP. It does so by presenting considerations for "what to monitor and manage" in an OLSRv2 network, and how. The approach developed is based on the Simple Network Management Protocol (SNMP), and thus this paper details the various Management Information Bases (MIBs) for router status monitoring and control - as well as a novel approach to history-based performance monitoring. While SNMP may not be optimally designed for MANETs, it is chosen due to it being the predominant protocol for IP network management - and thus, efforts are made in this paper to "adapt" the management tools within the SNMP framework for reasonable behavior also in a MANET environment.
\end{abstract}

Index Terms-OLSRv2, MANET, management, control, MIB, SNMP, performance monitoring

\section{INTRODUCTION}

Mobile Ad hoc Network (MANET) routing protocols are commonly assumed to be entirely self-managing: routers, running such a distributed protocol, perceive the topology of the MANET by means of control message exchange. Any change to the topology is reflected in the local routing tables of each router after a bounded convergence time, which allows forwarding of data traffic towards its intended destination. Usually, no human interaction is required, as all variable parameters required by the routing protocol are either negotiated in the control traffic exchange, or are only of local importance to each router (i.e. do not influence interoperability). However, external management and monitoring of a MANET routing protocol may be desirable to optimize parameters of the routing protocol. Such an optimization may lead to a more stable perceived topology and to a lower control traffic overhead, and therefore to a higher delivery success ratio of data packets, a lower end-to-end delay, and less unnecessary bandwidth and energy usage. This paper proposes a management framework to manage and control performance related objects on MANET routers running the Optimized Link State Routing Protocol version 2 (OLSRv2), which is currently in the process of being standardized by the MANET working group of the IETF ${ }^{1}$.

\footnotetext{
${ }^{1}$ The Internet Engineering Taskforce: http://www.ietf.org
}

\section{A. OLSRv2 Overview}

The Optimized Link State Routing Protocol version 2 (OLSRv2) [1], [2], [3], [4] is a successor to the widely deployed OLSR [5] routing protocol for MANETs. OLSRv2 retains the same basic algorithms as its predecessor, however offers various improvements, e.g. a modular and flexible architecture allowing extensions, such as for security, to be developed as add-ons to the basic protocol. OLSRv2 contains three basic processes: Neighborhood Discovery, MPR Flooding and Link State Advertisements. The basic operation of OLSRv2 is detailed in section I-A1 to I-A3 below, followed by a description of the flexible message format used by OLSRv2, in section I-A4, and a discussion of the configuration and operation of OLSRv2 routers in section I-A5.

1) Neighborhood Discovery (NHDP): The process, whereby each router discovers the routers which are in direct communication range of itself (1-hop neighbors), and detects with which of these it can establish bi-directional communication. Each router sends HELLOs, listing the identifiers of all the routers from which it has recently received a HELLO, as well as the "status" of the link (HEARD, verified bi-directional - called SYM). A router $a$ receiving a HELLO from a neighbor $b$ in which $b$ indicates to have recently received a HELLO from $a$ considers the link $a-b$ to be bi-directional. As $b$ lists identifiers of all its neighbors in its HELLO, $a$ learns the "neighbors of its neighbors" (2-hop neighbors) through this process. HELLOs are sent periodically, however certain events may trigger non-periodic HELLOs. NHDP enables each router interface to apply a hysteresis function which, in addition to the message exchange, may constrain when a link is considered as "usable" or not: for example, a router may elect to not consider, and thus not advertise, a link as SYM or HEARD unless a certain ratio of HELLOs are received, unless the SNR reaches a given threshold etc. Symmetrically, a router may decide to stop advertising a link as SYM or HEARD, subject to similar such constraints.

2) MPR Flooding: The process whereby each router is able to, efficiently, conduct network-wide broadcasts. Each router designates, from among its bi-directional neighbors, a subset (MPR set) such that a message transmitted by the router and relayed by the MPR set is received by all its 2-hop neighbors (i.e., the MPR set "covers" all 2-hop neighbors). MPR selection is encoded in outgoing HELLOs. The set of routers having selected a given router as MPR is the MPR- 
selector-set of that router.

3) Link State Advertisement: The process whereby routers are determining which link state information to advertise through the network. Each router must advertise links between itself and its MPR-selector-set, in order to allow all routers to calculate shortest paths. Such link state advertisements, carried in TC messages, are broadcast through the network using the MPR Flooding process. As a router selects MPRs only from among bi-directional neighbors, links advertised in TCs are also bi-directional. TC messages are sent periodically, however certain events may trigger non-periodic TCs.

4) Flexible Message Format: OLSRv2 employs the format specified in [1], for all protocol messages, thereby enabling scope-limited message flooding, compact (aggregated) address representation, also of non-contiguous network addresses, and the ability to associate any number of arbitrary attributes to either of control messages or addresses, by way of inclusion of Type-Length-Value objects (TLVs). The TLV structure permits any given message to be parsed correctly by allowing an implementation to "skip over" TLVs not recognized, thus enabling extensions to be developed that embed information into existing OLSRv2 control messages.

5) OLSRv2 Router Configuration: The configuration of an OLSRv2 router consists of the set of prefixes "owned", and thus advertised, by the router, as well as interfaces of that router, participating in the OLSRv2 routing protocol. For each such interface, a set of parameters apply; other than the IP address(es) of each interface, these parameters consist of control message emission intervals, as well as the hysteresis values and link quality estimation. It is important to note that agreement between OLSRv2 routers on the values for any of these is not required for interoperability. Link quality and hysteresis affect only which links a given router permits to become SYM or HEARD. Control message emission intervals and message content validity are encoded in outgoing control messages, by way of TLVs, such that a recipient router can process correctly these regardless of its own configuration.

\section{B. Paper Outline}

The remainder of this paper is organized as follows: Section II describes the motivation for the proposed SNMP-based management framework for OLSRv2-routed MANETs. The architecture of this framework is presented in section III. Section IV describes the construction and functioning of NHDP and OLSRv2 MIBs, while section V proposes the related REPORT-MIB - a convenient tool for performance management. This paper is concluded in section VI.

\section{Problem Statement}

As indicated in section I-A5, OLSRv2 imposes very minimal constraints on valid router configuration parameters, in order for OLSRv2 routers to interoperate. Fundamentally, the only parameter upon which agreement is required is $\mathrm{C}-\mathrm{a}$ constant, used to fix the scale and granularity of validity and interval time values, as included in protocol control messages. [2] proposes a value for this constant. As control messages carry validity time and interval time values, a recipient OLSRv2 router can behave appropriately, even if it uses vastly different values itself, as long as the recipient and sender use the same value for $\mathrm{C}$.
Link admittance, by way of the hysteresis values and link quality estimation, require no agreement; these are used for an individual router to determine a suitable threshold for "considering that a link could be a candidate for being advertised as usable".

Still, external monitoring and management may be desirable in an OLSRv2 network. A network may benefit from having its control message emission tuned according to the network dynamics: in a mostly static network, i.e. a network in which the topology remains stable over long durations, the control message emission frequency could be decreased in order to consume less bandwidth or less energy. Conversely, of course, in a highly dynamic network, the emission frequency could be increased for improved responsiveness. Concerning the hysteresis and link quality estimation, a management application might detect a region of an OLSRv2 network with a high link density - but also a high degree of "flapping": links coming "up" (SYM) only to disappear as LOST shortly thereafter. Detecting such behavior, on a global level and for multiple routers in the same region, could enable appropriately "tuning" the thresholds towards more stable links and, thus, a more stable routing structure in the network.

These are but two examples, and have as common that a more "global view" of the network, than that of a single OLSRv2 router, is required - i.e. entail that a Network Management System is able to inquire as to various performance values of the network, and to set various router parameters. Thus, a first-order task is to identify suitable management data for an OLSRv2 routed MANET, and to describe these by way of MIBs for use by an SNMP Network Management System.

In the following sections, the proposed MIBs for managing OLSRv2 networks and monitoring performance of these networks are described in detail.

\section{OLSRv2 MAnagement ARChitecture}

The proposed architecture of the OLSRv2 management system is depicted in figure 1. As is standard for SNMP management architectures, a Network Management System interacts with the various components of the device models directly over the network. However, frequent polling for object values in such a system involves a frequent and bandwidth-consuming message exchange. Further, due to highly variable network delays, it is not possible for a management application to determine the time associated with object values obtained via polling. In order to specifically address the issues associated with running SNMP for Performance Management over low bandwidth and high latency networks, typical of MANETs, the proposed Performance Management architecture is based upon a proxy capability, denoted REPORT-MIB [7]. This proxy is located in close proximity to the managed devices and offers remote generation of performance reports established via the management application using Remote Monitoring (RMON) style control and reporting. The proxy then polls (locally) for the current values of the relevant objects necessary for the generation of the performance reporting.

\section{NHDP AND OLSRV2 MIBS}

This section describes the design of the NHDP-MIB [8] and the OLSRv2-MIB [9]. As the protocols themselves are designed in a similar fashion, so are their associated MIBs. 


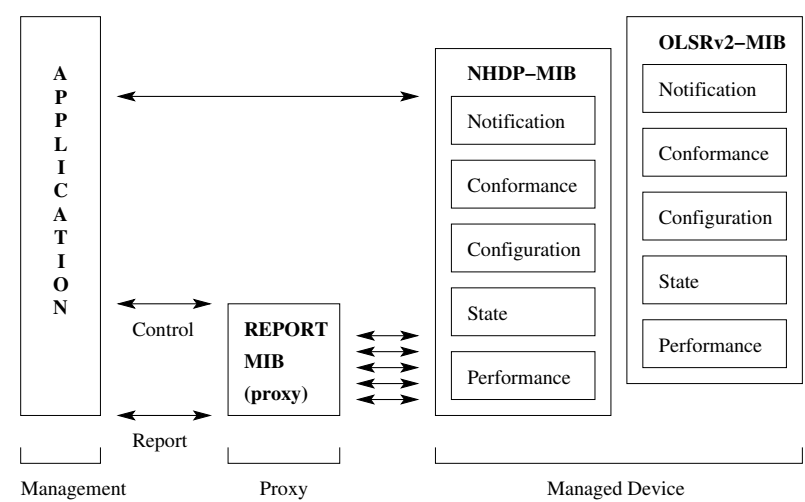

Fig. 1. The OLSRv2 management model

At the highest level, both the NHDP-MIB and the OLSRv2MIB are organized into the following groups:

- Configuration Group - switches, tables, objects which are initialized to default settings or set through the management interface defined by the MIB.

- State Group - automatically generated object values which define the current operating state of the NHDP or OLSRv2 protocol process in the router.

- Performance Group - automatically generated object values which help to assess the performance of the NHDP and OLSRv2 protocol.

- Notification Group - objects defining triggers and associated notification messages allowing for asynchronous tracking of pre-defined events on the managed device.

- Conformance Group - groupings of the above objects defining various compliance levels to the MIBs.

The Configuration Group for the NHDP-MIB and OLSRv2MIB includes objects which control message intervals (e.g. for HELLOs), information validity times (e.g. hold times), link quality (e.g. thresholds to determine usefulness of the links), and message jitter. For the OLSRv2-MIB, additional configuration information include objects related to hop limits and routers' willingness measures to act as MPRs. Details on the actions these objects have on the respective protocols are found in [3] and [4].

Regarding the State Group, both protocols are defined in terms of the various databases developed by the protocols in order for their proper function. The state tables in the OLSRv2 and NHDP MIBs are linked through two constructs (or TEXTUAL CONVENTIONS) developed within the MIBs as illustrated in figure 2. Within the NHDP and OLSRv2 protocol definitions, the various Information Bases provide information on discovered address sets, which are associated with discovered interfaces, which belong to discovered (or local) routers. The two TEXTUAL CONVENTIONS are used as indexes into the various State Tables of the NHDP-MIB. Further, as the related State Tables rely on the same indexing, it is relatively straightforward for a network management application to cross-reference data from the two MIBs.

Finally, the MIBs define two levels of Conformance; a Basic Compliance which includes only Configuration Group objects and a Full Compliance which includes Configuration, State, Performance and Notification Group objects.

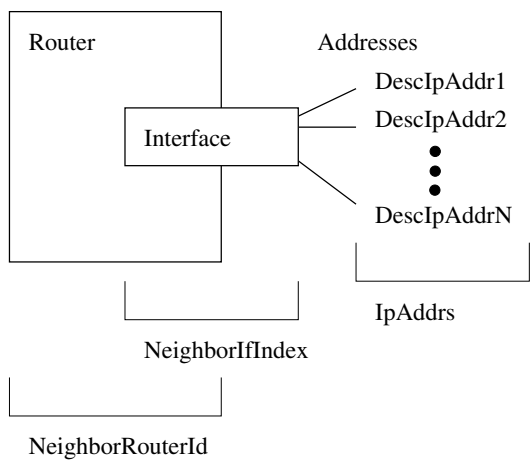

Fig. 2. The linkage between the OLSRv2 and the NHDP MIBs

\section{Performance Management}

Apart from objects for monitoring and controlling parameters and data sets in NHDP and OLSRv2 (specified in section IV), we propose a number of objects which permit operators to analyze the performance of NHDP and OLSRv2. This section describes the different types of objects and their intent for NHDP and OLSRv2.

\section{A. Object Types}

Some of the objects (denoted "base objects") explicitly appear in the NHDP-MIB and OLSRv2-MIB while others are obtainable through a combination of base objects from the MIB and reports available through the REPORT-MIB.

The full list of base objects in the NHDP-MIB and the OLSRv2-MIB is comprised of different counters (e.g. for counting the total number of transmitted HELLO messages, number of changes to the neighbor set and to the 2-hop set, etc.) and other object types.

In order to infer performance problems in an OLSRv2 network, it may not be sufficient to access base objects describing the total number of events, but objects describing the development of events over time. These objects, denoted "derived objects", are not specified in the NHDP-MIB and OLSRv2-MIB, but can be acquired using the REPORT- MIB. The REPORT-MIB allows operators to create reports "offline", possibly on another, more powerful device than the router running NHDP and OLSRv2. Notably, histories (based on timestamps) can be created over all of the performance related base objects.

For example, it is possible to create a histogram of intervals between transmitted HELLO messages, separated by periodic and triggered HELLOs. The histogram would display the distribution of intervals between two consecutive HELLOs of the same type (triggered or periodical) using a given bin size.

Moreover, the NHDP and OLSRv2 MIBs in combination with the REPORT-MIB allow operators to display the changes of the frequency by displaying the changes of histograms over time. The total duration of recorded events is split into a given number of equal bins. Then, a histogram is created for each bin and the "distances" are calculated between each two adjacent histograms in time (e.g. using the Bhattacharyya distance [10]). Note that while visualizing a change in the frequency of events may help the network administrator to understand changing properties of the network, it is out of scope of the MIB, and of this paper to automatically determine 
whether such a change indicates a performance problem or is part of the natural change of topology of the network.

\section{B. Derived Objects in NHDP and OLSRv2}

As described in section $\mathrm{V}-\mathrm{A}$, changes of the frequency of certain events may indicate performance issues in the MANET. Notably unstable neighbors or 2-hop neighbors and frequent changes of sets may have a negative influence on the performance of NHDP and OLSRv2, wherefore a number of derived objects have been specified in the MIBs that allow management applications to acquire information related to the stability of NHDP and OLSRv2. The following list describes several derived objects from the MIBs that are relevant for NHDP and OLSRv2 networks:

1) Frequency changes of message scheduling: A change in the message scheduling frequency can appear if, e.g., suddenly many triggered control messages are sent, whereas only few such triggered messages were sent in the past. This can indicate a sudden change in the topology perceived by a router.

2) Frequency changes of Neighbor Set modifications: Changes of frequency of neighbor-set modifications can indicate a performance problem. A neighbor-set modification is defined as a new neighbor that is added, a neighbor that is removed, or a neighbor that changes its symmetry status.

3) Frequency of changes of the online status of a neighbor: If a neighbor changes its "online" status very frequently (i.e. a neighbor tuple for that neighbor is alternatively added and removed again in a very short time), this may indicate a performance problem.

4) Frequency of changes of the online status of a 2-hop neighbor: Similar to the frequency of changes of the online status of a neighbor, an object in the MIB allows to track the frequency of change of the online status of 2-hop neighbors.

5) Frequency of changes of the link over which a neighbor is reachable: If a neighbor changes the interface over which it is reachable frequently, that can cause performance issues: (i) more in-router resources for updating the internal data structures, and (ii) additional control traffic messaging may be required (e.g. when sending triggered HELLO messages).

Such flapping of a neighbor may, for example, stem from inappropriate hysteresis values of the link quality selection of NHDP. Analyzing the frequency of neighbor flaps facilitates to modify the values to stabilize the link formation and removal on the OLSRv2 interfaces.

6) Frequency of changes of the neighbor over which a 2hop neighbor is reachable: Similar to the link-flapping of a neighbor as described above, a two-hop neighbor can flap between several one-hop neighbors, which may induce (i) routing set recalculation on all routers in the MANET, (ii) MPR set recalculations in the 2-hop neighborhood, and (iii) transmission of triggered control messages. The reason is that each time the 2-hop neighbor flaps between neighbors, a new MPR selection may be necessary.

7) Frequency of routing set recalculations and MPR set recalculations: The MIB provides two derived objects for observing routing-set and MPR-set recalculations over time. Both operations are costly in terms of in-router resources (such as memory and $\mathrm{CPU}$ time), and too frequent recalculations may reduce the life-time of the MANET when using batterypowered routers. The MIB objects allow an administrator to "tune" parameters of OLSRv2 in order to reduce the number of unnecessary recalculations.

\section{CONCLUSION}

The MANET routing protocol OLSRv2 does not require any external interaction once deployed, as routers are able to accommodate frequently changing network topologies in a self-organizing manner, as well as to accommodate OLSRv2 routers with heterogenous configuration in the same network. However, it is often desirable to monitor the network performance and to "tweak" parameters for improving the performance of an existing deployment of the routing protocol. This paper proposes a management and monitoring architecture for OLSRv2 routers based on SNMP, which allows a Network Management System (i) to acquire the state of the router (i.e. all parameters and information bases of the routing protocol), (ii) to modify parameters during runtime, and (iii) to generate offline performance reports. As for (i) and (ii), two Management Information Bases (MIBs) are proposed for OLSRv2 and for the neighborhood discovery part of OLSRv2, called NHDP. (iii) is derived through the creation of an external proxy service, the REPORT-MIB, typically running on the same machine as the agents (exposing the information defined by the OLSRv2-MIB and NHDP-MIB). The rationale is to avoid frequent polling over the network, leading to a frequent and bandwidth-consuming message exchange.

This paper specifies a number of performance reports that concern the stability of the nearby topology of a router. When some of the router parameters in OLSRv2 and NHDP (such as the link quality related parameters) are unwisely set with respect to the characteristics of a given network, the local topology may "flap" between several possible configurations, thus leading to additional control traffic overhead, in-router calculations and deteriorated performance. Detecting such behavior, on a global level and for multiple routers in the same region, could enable appropriately "tuning" the parameters towards a more stable routing structure in the network.

\section{REFERENCES}

[1] T. Clausen, C. Dearlove, J. Dean, C. Adjih, "RFC5444: Generalized Mobile Ad Hoc Network (MANET) Packet/Message Format", Std. Track, http://www.ietf.org/rfc/rfc5444.txt

[2] T. Clausen, C. Dearlove, "RFC5497: Representing Multi-Value Time in Mobile Ad Hoc Networks (MANETs)", Std. Track, http://www.ietf.org/rfc/rfc5497.txt

[3] T. Clausen, C. Dearlove, J. Dean, "I-D: MANET Neighborhood Discovery Protocol (NHDP)", Work In Progress, http://tools.ietf.org/id/draft-ietfmanet-nhdp-14.txt

[4] T. Clausen, C. Dearlove, P. Jaquet, "I-D: The Optimized Link State Routing Protocol version 2 (OLSRv2)", Work In Progress, http://tools.ietf.org/id/draft-ietf-manet-olsrv2-11.txt

[5] T. Clausen, P. Jacquet, "RFC3626: Optimized Link State Routing Protocol (OLSR)", Experimental, http://www.ietf.org/rfc/rfc3626.txt

[6] D. Levi, P. Meyer, B. Stewart, "RFC3413: Simple Network Management Protocol (SNMP) Applications", Std. Track, http://www.ietf.org/rfc/rfc3413.txt

[7] R. G. Cole, J. Macker, A. Morton, "I-D: Definition of Managed Objects for Performance Reporting", Work in Progress, http://tools.ietf.org/id/draft-ietf-manet-report-mib-00.txt

[8] U. Herberg, R. Cole, I. Chakeres, "I-D: Definition of Managed Objects for the Neighborhood Discovery Protocol", Work In Progress, http://tools.ietf.org/id/draft-ietf-manet-nhdp-mib-04.txt

[9] U. Herberg, R. Cole, T. Clausen, "I-D: Definition of Managed Objects for the Optimized Link State Routing Protocol version 2", Work In Progress, http://tools.ietf.org/id/draft-ietf-manet-olsrv2-mib-02.txt

[10] A. Bhattacharyya, "On a measure of divergence between two statistical populations defined by their probability distributions", Bull. Calcutta Math. Soc. 35, 99-109, 1943 